\title{
Chillax surgery: back to the roots
}

\author{
F. M. Riegler
}

Published online: 6 December 2012

(C) Springer-Verlag Wien 2012

\section{Dear readers,}

The ancient mankind did not have a problem to express knowledge in the form of images. Thus, sculptures, paintings, myths pictured the experiences and fused the academic and metaphysic world into one. Today, we have gained enormous scientific knowledge at the cost of dream, phantasy and imagination $(p<0.001)$ (Fig. 1). Going in line with the notion, the ancient cultures fostered the believe that man originated from the stars and after death returns back to the stars to become a star. Thus, the being on earth was densely connected to the universe. Therefore, in the old myths man and universe were one, the world was perceived as the numerous and almost endless representations of the essence, the do, the will, the pushing purusha power, the Hebrew quone (first and only appearance in the socio-religiouseconomic context of the "messiah" of Abraham by the well-paid priest Melchizedek), the driving force (I. Newton), the self creator, the self re-newer experience. Following the hundred thousands of years of integrative imagination, before the birth of the physics and metaphysics (are is tot el es), a Seth came and dissected the Osiris into 72 accurate pieces, which were spread all over the Milky Way river Nile. Now Isis will have to come for the interdisciplinary repair of the Osiris, the knowledge of the lost civilization before, during and after the last ice age (we go for a global warming). Here surgery comes into play: connect to function. Remains to be questioned the impact of the above considerations for every day surgery.

Surgery happens in the operation theatre (applause!), ages of stages for wages of a wide spectrum of personalities ranging from the archaic worship leader outlaw hard rocker to the hypersensitive self-destructing dissolver. Herein, the fire burns out under the light of the ancestors'

\footnotetext{
F. M. Riegler, MD $(\bowtie)$

Manometry Lab and Department of Surgery,

Medical University of Vienna, Waehringer Guertel 18-20, 1090 Vienna, Austria

e-mail: Franz.Riegler@meduniwien.ac.at
}

fathers and mothers and leads to the inability to get rid of the ridges of life and guides an increasing number of individuals towards the shake down soul party within the office room of an almighty brilliant cash the trash therapeutic preacher teacher innovator. The reincarnated eagle landed and expressed itself in the form of organic dysfunction, disease and the inability to counteract the impairment of life quality, productivity and well-being (WHO definition of disease: http://www.youtube.com/ watch?v=nTKVq3V6qNU). Moon, stars, suns and planets of the outer world reside within the representation points of the body and form the milky way of dreams, desires and hopes. In addition, here alternative strategies come into play, needles and fingers are pushed, pulled and tracked down to the bone.

In other words, man needs time and space, care and attention, may it be due to the lack of love? We have to invest more time to listen to the patient, before we start to act. The listen and learn cannot replace any test including the amazing high tech orchestra ranging from computed tomography to magnetic resonance imaging, from in vivo macro microscopy high definition endoscopy to immunohistochemistry, from genetics to polymerase chain reaction. It is time for the physicians to stand up and claim for adequate salary for taking the patient history: this will definitely save expenses, unnecessary tests and treatments. You cannot make the job in 2-5 min. If you invest more time, you will crash. The system does not care about a sinking ship, no wave points to the sunken souls. Most importantly, we have to live the awareness that concentrated sugar represents the most effective chemotherapy that poisons man, destroys the capability for effective reasoning and is the cause for almost all internal diseases. Furthermore, metabolically distorted politicians suffering from hypertension, diabetes, coronary heart's disease, obesity, arthritis, migraine decide on the destiny of our continent (sic!). Sugar causes cancer. Due to their manifestations of the dysbalance of their metabolism, these individuals are forced to become the boilers of the drugs, which aim to treat the side effects of the side effects of the side effect drugs: they poison 


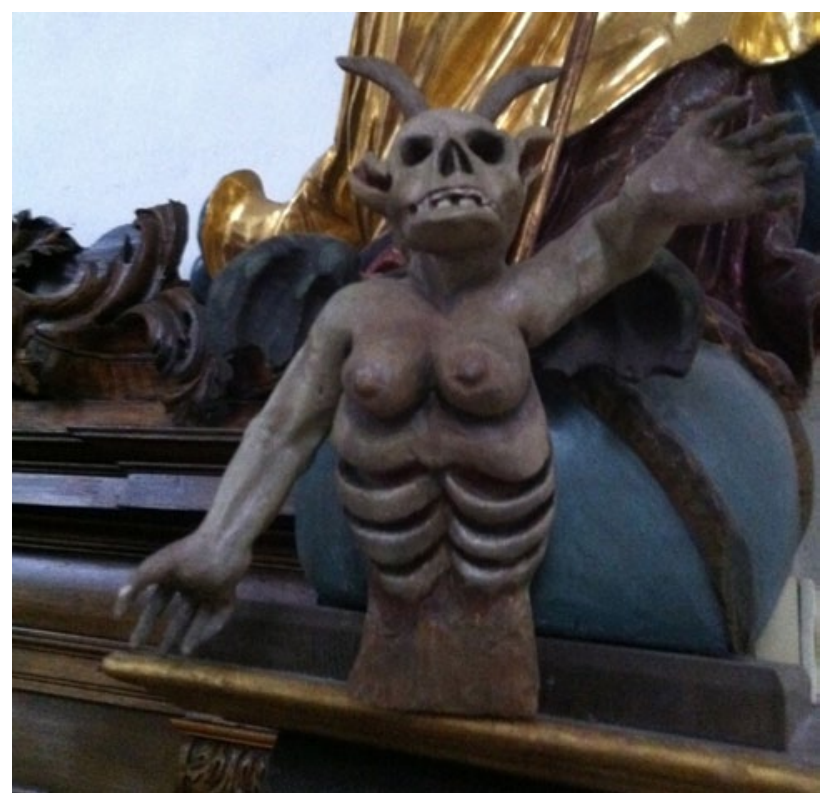

Fig. 1 Female devil sixteenth century, wood: fusion of physics and metaphysics creates a specific state of mood which mirrors the idea of the author, that today we have lost dream at the cost of knowledge. It also mirrors the seduction of man by the products of the food industry. Image taken by the author at the church of Zell am Pettenfirst, Upper Austria, Europe, using iPhone technology

themselves within the postprandial hypoglycemic shock waves of their alcoholic and carbohydrate democracy. Due to the greed-driven irresponsibility of the politicians and the food industry, the Europeans are addicted to sugar and experience the stratospheric bull of the coke metabolism (Fig. 1). As a consequence, politicians are what they are: metabolic-disordered sick females and males. Europe eats itself up. Africa is waiting to come and take over. They will find food starving obese Portuguese, Italians, Greeks, Spanish, French and Croatians' French fries consumers stuck in the sand, looking like the Easter Island Moai (who the hell fixed them there?) [1], they will find stroke-rocked hobblers resembling the futuristic central, northern, western and eastern European inhabitants of the pictures of $\mathrm{H}$. Bosch [2]. We have to stop eating too much, too often, and too sweet. We have to stop to tell us lies. We have to accept: nutrition needs a change; we have to inform the public, the consumers. Otherwise, we will be burned out within the fire of the profit of the

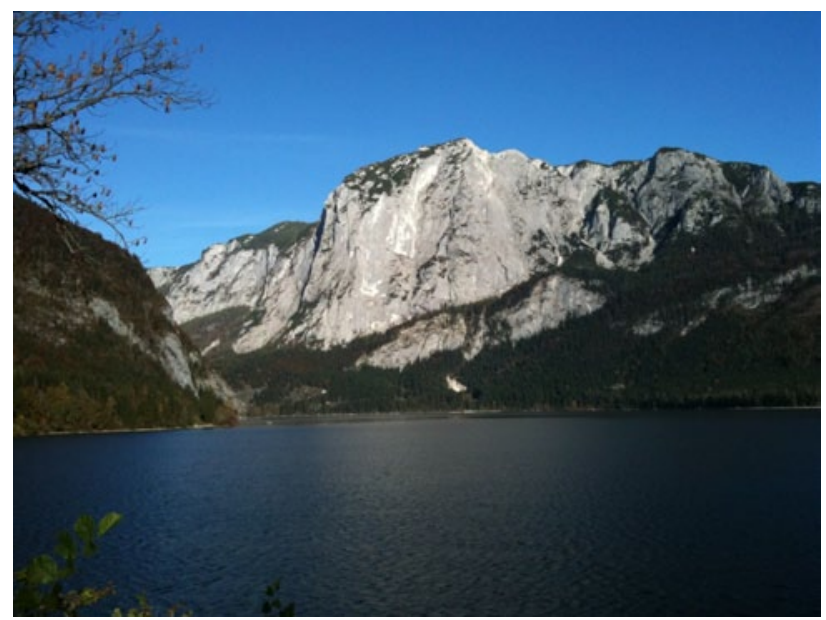

Fig. 2 Nature energy attracts, it reminds us on our origins and the need for a quiet place to chillax from the greed-driven overdo of the modern civilization. The image mirrors the idea of the authors, that from time-to-time we should go back to the roots. Image taken by the author in Altaussee, Styria, Europe, using iPhone technology

food industry. At the end, no consumer may be left and the parasite will die with the host.

Let us return to the roots (Fig. 2), from time-to-time, let us tell stories about the human glories, where star becomes man and man becomes a star, where art represents formed love, where man finds in utero security and cosiness and fosters phantasy. At nighttime, raise your heart, lift your head and look up into the skies. Allow you, be you, relax and stay tuned. Seasonal greetings!

\section{Conflict of interest}

The author declares that there exists no conflict of interest.

\section{References}

1. Lipo CP, Hunt TL, Rapu Haoa S. The "walking" megalithic statues (moai) of Easter Island. J Archaeol Sci. 2012. Epub ahead of print.

2. Vander Kooi C. Hieronymus Bosch and ergotism. WMJ. 2012;111(1):4. 